\title{
MÉTODO DE REDUCCIÓN DE MUESTRA PARA ENSAYOS DE LABORATORIO EN MEZCLA ASFÁLTICA EN CALIENTE
}

\section{SIZE REDUCTION METHOD FOR LABORATORY TESTING IN HOT MIX ASPHALT}

Ing. Fabián Elizondo Arrieta, MBA

Coordinador de Laboratorios de Infraestructura Vial

LanammeUCR, Costa Rica

E-mail: fabian.elizondo@ucr.ac.cr

\section{RESUMEN}

Para el año 2004 no se contaba con métodos de reducción de muestra para mezcla asfáltica en caliente estandarizados y se tomaban como referencia los métodos de cuarteo para agregados pero estos no indicaban obviamente la temperatura a la cual se debía calentar la muestra para asegurar su trabajabilidad y que no afectara las propiedades de la mezcla entre otros detalles. Es por esta razón que, en el LanammeUCR se implementó un método totalmente nuevo para poder certificar el muestreo de mezcla asfáltica en caliente en vagoneta, por lo que se aprovechó este estudio para incluir el método de cuarteo para demostrar que las submuestras son representativas del lote de muestra.

Se realizó un diseño experimental el cual incluía la realización del método de cuarteo para varias cajas de mezcla para luego determinar la granulometría y el contenido de asfalto con el ensayo de ignición para cada submuestra, así como el ensayo de gravedad específica máxima teórica, con lo cual se demostró que con este método sí es posible obtener submuestras representativas del lote con buena repetibilidad, se comprobó que la segregación de la mezcla se eliminó con el uso de la canaleta y además de que se obtienen las submuestras deseadas en un solo paso.

Este método tiene 11 años de implementado y las ventajas sobre el método estándar AASHTO R 47 publicado en el año 2008 es que, en este método se detallan los cuidados que se tienen que tomar en cuenta a la hora de manipular la mezcla asfáltica en caliente, tales como las temperaturas a las que se tiene que calentar la muestra y los tiempos de calentamiento, pues esto afecta de manera significativa las propiedades del material. Además se incluye el uso de herramientas manuales muy simples que son de fácil fabricación y económicas que tomaron encuenta el criterio de ergonomía para asegurar que el procedimiento fuese seguro y práctico.

\section{PALABRAS CLAVE:}

Muestra, submuestra, reducción de muestra, ensayo de laboratorio, mezcla asfáltica en caliente
Ing. Mónica Jiménez Acuña

Jefe de Laboratorio de Ensayos Dinámicos para Pavimentos

LanammeUCR, Costa Rica

E-mail: monica.jimenez@ucr.ac.cr

\section{ABSTRACT}

In 2004 there were not available standardized methods of reducing a large sample of hot mix asphalt and the quartering methods for aggregates were taken as reference, but these do not take into account the temperature at which the mix should be heated to ensure workability and the way to not affect the properties of the mixture within other details. It is for this reason that LanammeUCR implemented a completely new method to be able to certify the sampling of hot mix asphalt in the truck transports, so this study was used to include the quartering method to show that the sub-samples are representative of the lot.

An experimental design was developed which included the new quartering method for multiple sampling boxes and then measure the asphalt content and gradation with the ignition test for each subsample and also the maximum theoretical specific gravity was performed, it was shown that with this method it is possible to obtain representative subsamples with good repeatability and also found that the segregation of the mixture was eliminated using the gutter system that in one step the desired subsamples are obtained.

This method has 11 years of implementation and the advantages over the standard method AASHTO R 47, published in 2008, is that in this approach the care that must be taken when handling hot mix asphalt are detailed, such as the temperatures at which the sample must be heated and the heating times are established as this significantly affects the material properties. Besides the use of simple hand tools that are easy and economical to manufacture they take into account an ergonomic criteria to ensure that the procedure was safe and practical.

\section{KEYWORDS:}

Sample, subsample, size reduction, laboratory testing, hot mix asphalt 


\section{INTRODUCCIÓN}

Para el año 2004 no se contaba con métodos de reducción de muestra para mezcla asfáltica en caliente estandarizados y se tomaban como referencia los métodos de cuarteo para agregados pero estos no indicaban obviamente la temperatura a la cual se debía calentar la muestra para asegurar su trabajabilidad y que no afectara las propiedades de la mezcla entre otros detalles.

Es por esta razón que, el LanammeUCR implementó un método totalmente nuevo para poder certificar el muestreo de mezcla asfáltica en caliente en vagoneta, por lo que se aprovechó este estudio para incluir el método de cuarteo para demostrar que las submuestras son representativas del lote de muestra.

El método de reducción con cuarteador mecánico expuesto en el método AASHTO es poco práctico pues se tiene que calentar los cuarteadores los cuales son grandes y esto produce que se tengan que utilizar hornos grandes y también produce que el manejo de los equipos sea complicado para evitar quemaduras, también este método se tiene que repetir las veces que sea necesario hasta obtener el tamaño de muestra deseado y no toma en cuenta el enfriamiento de la mezcla y el tiempo que conlleva hacer esto, lo que afecta de manera significativa la homogeneidad de las submuestras pues al inicio cuando la mezcla está más caliente el ligante asfáltico se adhiere a las paredes con la consiguiente pérdida del material (aún cuando indica que hay que utilizar un antiadherente), para cuando la muestra se enfría este fenómeno no se presenta pero la mezcla se aglomera en las partes finas lo que hace que la homogeneidad se vea afectada.

Cuando se trató de implementar este método con el cuarteador grande, la mezcla se enfrió rápidamente y por la cantidad de peso que se introdujo, cuando se abrieron las compuertas del cuarteador, la mezcla no cayó en las bandejas. Por lo tanto, se demostró que este procedimiento era poco práctico.

Los cuarteadores pequeños no sirven para el tamaño de muestras que normalmente se muestrea (peso de $21 \mathrm{~kg}$ ), pues no cabe en la bandeja del cuarteador. Por lo que no es posible utilizar este método para muestras de gran tamaño.

Los métodos de reducción tipo incremental tienen el problema que indican que se tiene que hacer un acomodo de la mezcla en un apilamiento tipo cónico, lo que hace que el agregado grueso se separe y produce una clara segregación de la muestra a pesar que el mismo método indica que se tiene que evitar, pero los agregados de gran tamaño y peso siempre van a tender a segregarse, sobre todo en mezclas asfálticas de graduación abierta.
Es de interés que este método sea conocido y aplicado en otros laboratorios de autocontrol, control y verificación de la calidad de la mezcla asfáltica en caliente a nivel nacional, para uniformizar los métodos más aún que están demostradas las debilidades del método AASHTO.

\section{OBJETIVO}

Implementar un método de ensayo nuevo que fuera práctico, fácil de aprender y que produjera que las submuestras sean representativas del lote. Sobre todo para muestras de mezcla asfáltica grandes entre $15 \mathrm{~kg}$ y $25 \mathrm{~kg}$.

\section{METODOLOGÍA Y RESULTADOS}

\subsection{Antecedentes}

El método de cuarteo para mezcla asfáltica en caliente utilizado antes del año 2004 era un procedimiento llamado el cuarteo Tipo B, el cual consistía en homogenizar la muestra en un apilamiento tipo cónico y luego con ayuda de un dispositivo para enrasar grande se reduce su altura hasta obtener un círculo, el cual luego se separaba en cuartos y cada cuarto se separaba de igual manera en porciones tales que al combinar dos opuestas formaran la submuestra, tal como se explica en el método B del estándar AASHTO T 248 que se muestra en la Figura 1.

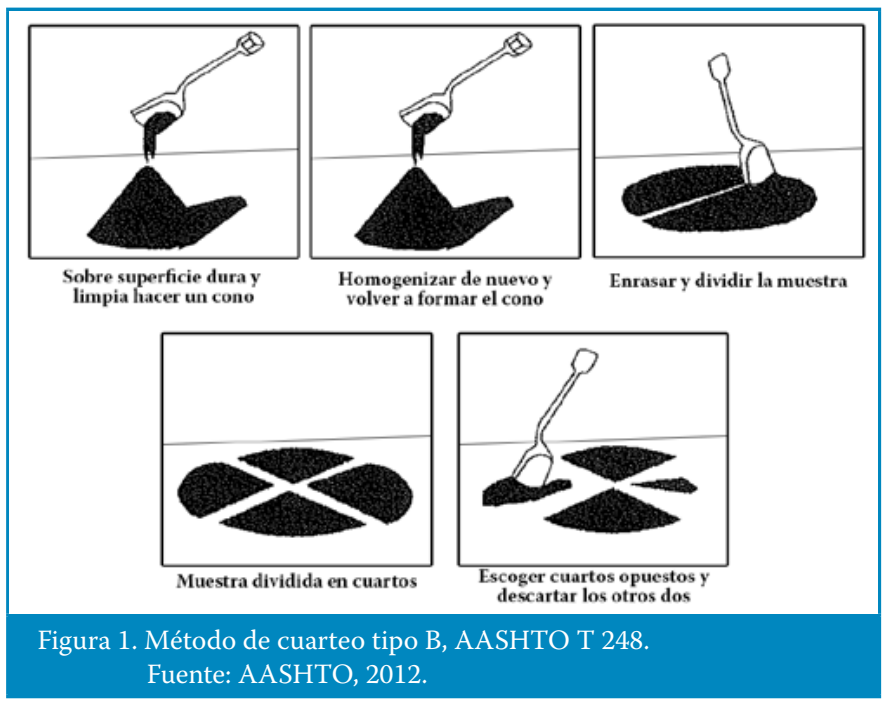

Para este método era muy claro que se presentaba segregación de la muestra, sobre todo con materiales muy gruesos, donde el agregado más grande rodaba hacia abajo a la hora de hacer el apilamiento cónico. Adicionalmente como no estaba establecida la temperatura a la cual calentar las muestras, se utilizaba una temperatura de $110^{\circ} \mathrm{C}$, la cual se estableció por experiencia y tratando de tomar en cuenta que la mezcla no sufriera sobrecalentamiento para no ocasionar oxidación excesiva. 
Por lo que para establecer si verdaderamente existía la segregación de la muestra se obtuvieron 10 submuestras para el ensayo de contenido de asfalto por ignición y también se determinó la granulometría de los agregados extraídos, para establecer estadísticamente si el fenómeno era despreciable y no tener que cambiar el método de reducción.

Luego de estudiar los resultados obtenidos del mismo lote, se pudo observar que no era posible cumplir con los límites permitidos de variación para las propiedades: contenido de asfalto, porcentajes pasando el tamiz de $9,5 \mathrm{~mm}\left(\mathrm{~N}^{\circ} 3 / 8\right)$, tamiz de $4,75 \mathrm{~mm}\left(\mathrm{~N}^{\circ} 4\right)$, y tamiz de $0,075 \mathrm{~mm}\left(\mathrm{~N}^{\circ} 200\right)$. Los resultados se presentan en la siguiente Figura 2.

Como se puede observar de la Figura 2, los valores obtenidos para la mayoría de las submuestras presentan una alta variabilidad e incluso también no se cumple con la diferencia máxima aceptable para los tamices clave de una granulometría de mezcla asfáltica en caliente. Esto se puede observar de acuerdo con los límites de control superior e inferior (LCS y LCI) de cada parámetro calculados de acuerdo con el control estadístico del proceso. Dados estos resultados no se podía pretender legitimar el método pues había una alta variabilidad, por lo tanto, se tuvo que pensar en un nuevo método para eliminar las posibles fuentes de errores sistemáticos.

Se estudiaron varias fuentes internacionales pues para ese tiempo todos los laboratorios utilizaban el método de cuarteo Tipo B. El primer intento fue utilizar el cuarteador mecánico, pues varias de las fuentes aplicaban este método. Pero dada la poca experiencia que se tenía con respecto a su uso para mezclas se presentaron varios inconvenientes, pero el más destacado fue que se perdió mucha temperatura a la hora de manipular la muestra y no fue posible que esta pasara por el espacio del embudo y más bien toda la muestra quedó atorada.

Otra desventaja del método $B$ es que una muestra de $20 \mathrm{~kg}$ se tenía que separar en 12 porciones para obtener una submuestra para el ensayo Marshall, lo que hacía además que estas porciones no fueran homogéneas por lo estrechas que eran. Adicionalmente, en un cuarteo también había que sacar más tipos de muestras para los distintos ensayos, lo que hacía al proceso largo y tedioso.

Fue en este punto que definitivamente había que pensar en un nuevo método más práctico, rápido y que no fuera tan complejo de aplicar.
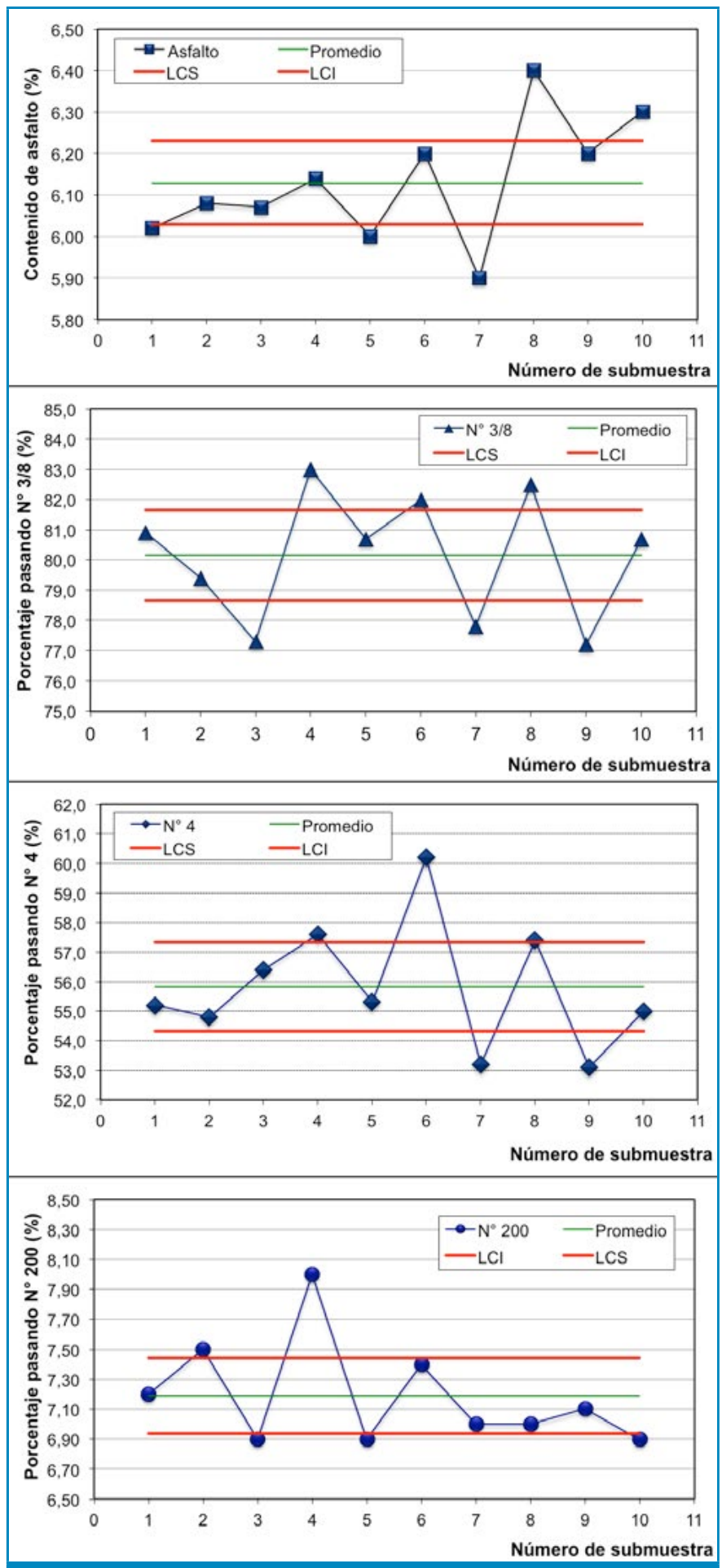

Figura 2. Comportamiento del método de cuarteo Tipo B.

\subsection{Propuesta de la nueva metodología}

Lo primero que se hizo fue establecer la temperatura de calentamiento de la muestra, se propuso una temperatura de $110^{\circ} \mathrm{C}$, la cual ya se utilizaba y que no es tan alta para envejecer la mezcla y no es tan baja como para que los periodos en el horno fueran extensos, esto se hizo a prueba y error. Se encontró que ya a las 3 horas en el horno una muestra entre $20 \mathrm{~kg}$ y $30 \mathrm{~kg}$ se encontraba en un estado suelto y trabajable. 
Lo siguiente que se estableció fue no hacer el apilamiento cónico para eliminar del todo la posibilidad de segregación de las partículas gruesas, por lo que se propuso hacer la homogenización de la muestra de la siguiente manera: al homogenizar la muestra se debe procurar que el material quede dispuesto en forma rectangular (más parecido a un camellón), la utilización del separador pequeño puede ser necesaria para acercar las partículas dispersas para terminar de conformar el rectángulo sin que este se segregue (Ver Figura 3).

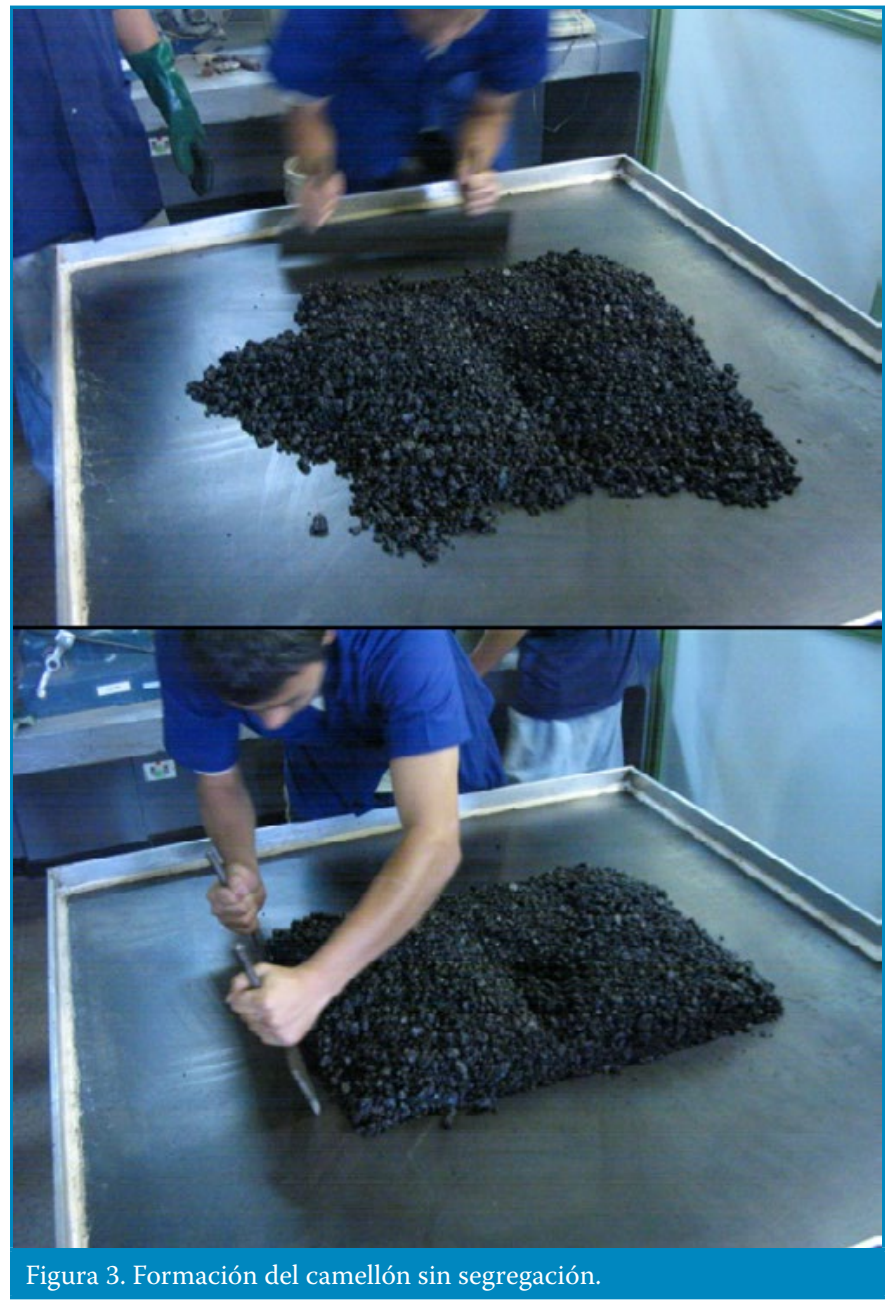

Es en este punto que se añade la innovación, pues se tenía que idear cómo obtener las submuestras en un solo momento, por lo que se inventó utilizar una canaleta metálica para evitar la segregación a la hora de sacar las mismas.

Con el rectángulo de mezcla conformado y con la ayuda de la pala de muestreo y el separador grande se tomaron porciones de material, las cuales fueron dispuestas a lo largo del canal de metal. Como se muestra en la Figura 4 el separador grande se utiliza para separar franjas de material que serán recogidas con la pala de cuarteo y depositadas una al lado de la otra en la canaleta, iniciando en el extremo desprendible del canal y finalizando en el extremo fijo.

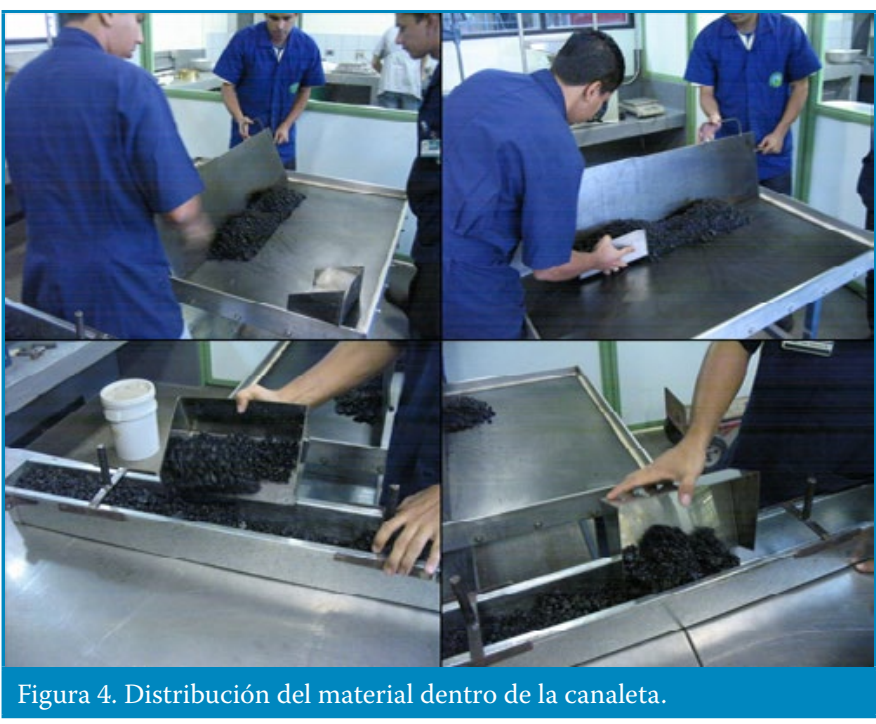

Una vez dispuesto el material dentro del canal de metal se introduce un aislador de metal, a una distancia del extremo removible del canal que dependerá de la cantidad de material necesaria para conformar el objeto de ensayo. Una vez hecho esto, se desprende el extremo removible y se desplaza el aislador uno hasta el extremo descubierto, depositando el material en un tazón que se coloca sobre una balanza (Ver Figura 5).

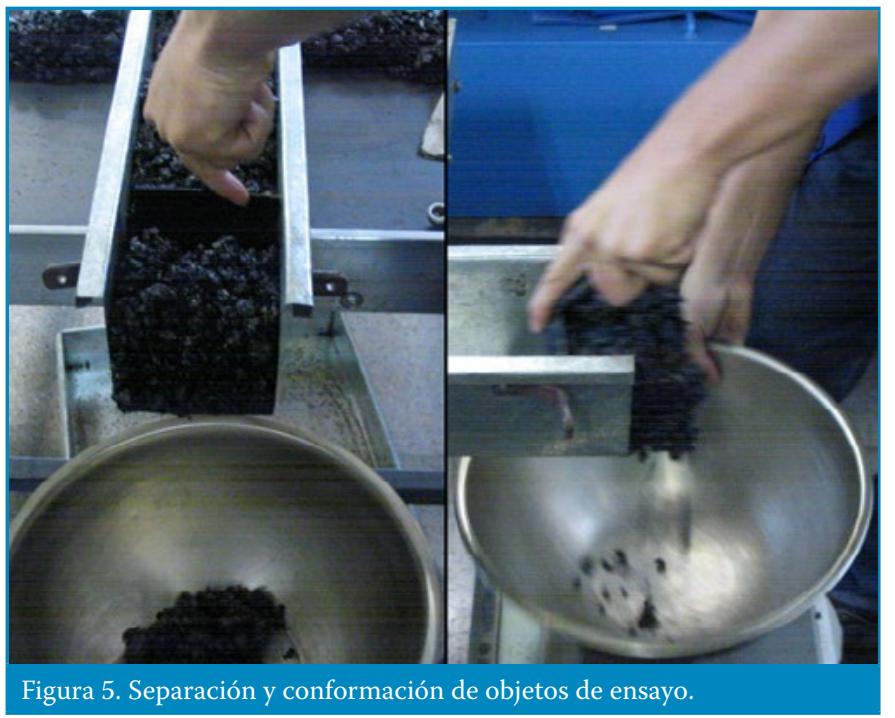

Una vez que se implementó el ensayo se procedió a validar los resultados que se obtienen con el nuevo método produciendo la mezcla asfáltica en el laboratorio. Se produjeron dos muestras de $18 \mathrm{~kg}$ cada una y se procedió luego a cuartearlas para obtener 8 submuestras para el ensayo de contenido de asfalto por ignición y la granulometría del agregado extraído. Una vez obtenidos los resultados de cada ensayo se procedió a realizar un análisis estadístico de los datos utilizando el análisis de varianza de un factor (ANOVA). 


\subsection{Comprobación de la metodología propuesta}

Como en todo análisis de este tipo, la hipótesis nula es que las dos muestras vienen de la misma población. El nivel de confianza utilizado fue de un $95 \%$ para un $\alpha=0,05$. Se analizaron las variables contenido de asfalto y todos los tamices que conforman una granulometría de mezcla asfáltica. Por ejemplo, se muestra en la Tabla 1 los resultados del análisis de varianza para cada variable.

\begin{tabular}{|c|c|c|c|c|c|}
\hline Variable & $\begin{array}{l}\text { Fuente de } \\
\text { Variación }\end{array}$ & GL & $\begin{array}{l}\text { Suma de } \\
\text { Cuadrados }\end{array}$ & $\begin{array}{c}\text { Media } \\
\text { Cuadrática }\end{array}$ & Prob $>$ F \\
\hline \multirow{3}{*}{$\begin{array}{l}\text { Contenido } \\
\text { de asfalto }\end{array}$} & MAC & 1 & 0,0315 & 0,03151 & 0,2409 \\
\hline & Error & 14 & 0,2940 & 0,02100 & \\
\hline & C. Total & 15 & 0,3255 & & \\
\hline \multirow{3}{*}{ Tamiz 3/4 } & MAC & 1 & 0,0000 & 0,00001 & 0,3343 \\
\hline & Error & 14 & 0,0001 & 0,00001 & \\
\hline & C. Total & 15 & 0,0001 & & \\
\hline \multirow{3}{*}{ Tamiz 1/2' } & MAC & 1 & 0,4660 & 0,46596 & 0,7449 \\
\hline & Error & 14 & 59,2251 & 4,23037 & \\
\hline & C. Total & 15 & 59,6911 & & \\
\hline \multirow{3}{*}{ Tamiz 3/8' } & MAC & 1 & 1,0507 & 1,05072 & 0,7496 \\
\hline & Error & 14 & 138,7790 & 9,91278 & \\
\hline & C. Total & 15 & 139,8297 & & \\
\hline \multirow{3}{*}{ Tamiz $\mathrm{N}^{\circ} 4$} & MAC & 1 & 0,3730 & 0,37303 & 0,7968 \\
\hline & Error & 14 & 75,7919 & 5,41371 & \\
\hline & C. Total & 15 & 76,1650 & & \\
\hline \multirow{3}{*}{ Tamiz No 8} & MAC & 1 & 0,3520 & 0,35198 & 0,6275 \\
\hline & Error & 14 & 20,0226 & 1,43018 & \\
\hline & C. Total & 15 & 20,3745 & & \\
\hline \multirow{3}{*}{$\begin{array}{c}\text { Tamiz } \mathrm{N}^{\circ} \\
16\end{array}$} & MAC & 1 & 0,7702 & 0,77021 & 0,2216 \\
\hline & Error & 14 & 6,5881 & 0,47058 & \\
\hline & C. Total & 15 & 7,3583 & & \\
\hline \multirow{3}{*}{$\begin{array}{c}\text { Tamiz } \mathrm{N}^{\circ} \\
30\end{array}$} & MAC & 1 & 0,1168 & 0,11678 & 0,4827 \\
\hline & Error & 14 & 3,1435 & 0,22453 & \\
\hline & C. Total & 15 & 3,2602 & & \\
\hline \multirow{3}{*}{$\begin{array}{c}\text { Tamiz } \mathrm{N}^{\circ} \\
50\end{array}$} & MAC & 1 & 0,0629 & 0,06292 & 0,5011 \\
\hline & Error & 14 & 1,8469 & 0,13192 & \\
\hline & C. Total & 15 & 1,9098 & & \\
\hline \multirow{3}{*}{$\begin{array}{c}\text { Tamiz N}^{\circ} \\
100\end{array}$} & MAC & 1 & 0,0379 & 0,03792 & 0,4955 \\
\hline & Error & 14 & 1,0839 & 0,07742 & \\
\hline & C. Total & 15 & 1,1218 & & \\
\hline \multirow{3}{*}{$\begin{array}{c}\text { Tamiz N }^{\circ} \\
200\end{array}$} & MAC & 1 & 0,0388 & 0,03878 & 0,3678 \\
\hline & Error & 14 & 0,6269 & 0,04478 & \\
\hline & C. Total & 15 & 0,6656 & & \\
\hline
\end{tabular}

De la Tabla 1 se concluye que no hay diferencias significativas entre las muestras, por lo tanto se puede inferir que el método de cuarteo es repetible y que se obtienen resultados aceptables. Además, el problema de segregación de la mezcla se eliminó.

Adicionalmente se realizó la prueba estadística de comparación de promedios de Tukey-Kramer HSD para asegurar las conclusiones. En la Figura 6 se presenta el resultado obtenido para el contenido de asfalto donde visualmente se puede observar que los promedios no difieren.

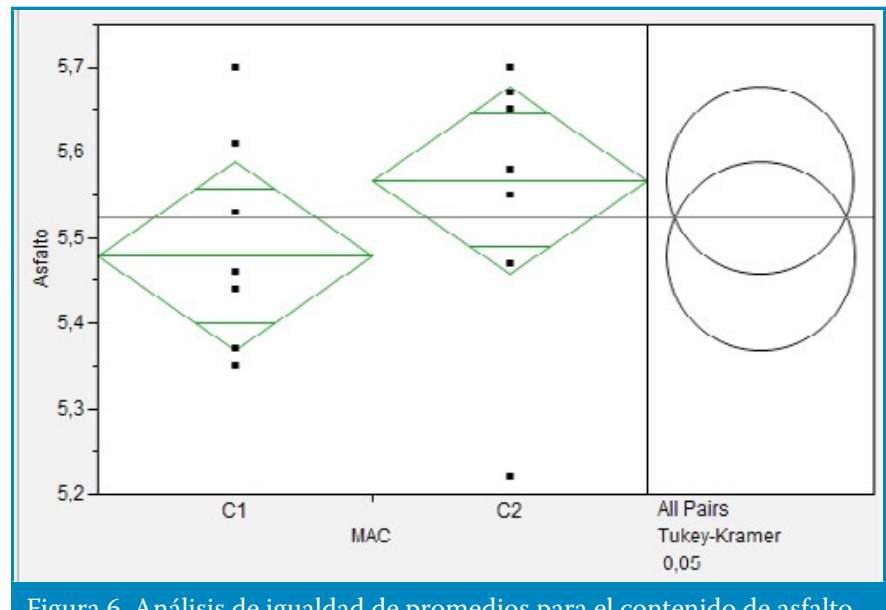

Finalmente, como era una mezcla preparada en el laboratorio, también se analizó qué tan lejano estaba el resultado obtenido en el ensayo del valor verdadero, por ejemplo para el contenido de asfalto las muestras se prepararon con un contenido de 5,5\% sobre peso total de mezcla. En la Tabla 2 se muestra el resultado obtenido del cual podemos concluir que las dos muestras no se alejan del valor verdadero dado que la probabilidad es mayor al valor alfa $\alpha=0,05$.

\begin{tabular}{|r|l|}
\hline \multicolumn{2}{|c|}{ Test Mean=value } \\
\hline Valor hipotético & 5,5 \\
\hline Estimado real & 5,52313 \\
\hline GL & 15 \\
\hline Desviación estándar & 0,14732 \\
\hline & Prueba t \\
\hline Estadístico & 0,6279 \\
\hline Prob $>$ Itt & 0,5395 \\
\hline Prob $>\mathrm{t}$ & 0,2698 \\
\hline Prob $<\mathrm{t}$ & 0,7302 \\
\hline Tabla 2. Resultados del análisis de igualdad al valor verdadero \\
para el contenido de asfalto.
\end{tabular}




\section{CONCLUSIONES Y RECOMENDACIONES}

De todos los análisis realizados, se puede concluir que este nuevo método para la separación de muestras de mezcla asfáltica en caliente fue validado y que es aceptable y produce resultados adecuados, por lo que el LanammeUCR tiene ya más de 10 años de estarlo utilizando.

El método es sencillo y muy práctico.

Las herramientas a utilizar son de fácil construcción y la canaleta utilizada se puede conseguir con facilidad en cualquier ferretería.

El método de reducción utilizando cuarteador mecánico expuesto en el método AASHTO es poco práctico pues se tiene que calentar los cuarteadores los cuales son grandes y esto produce que se tengan que utilizar hornos grandes y también genera que el manejo de los equipos sea complicado, esto con el fin de para evitar quemaduras. Adicionalmente este método se tiene que repetir las veces que sea necesario hasta obtener el tamaño de muestra deseado y no toma en cuenta el enfriamiento de la mezcla y el tiempo que conlleva hacer esto, lo que afecta de manera significativa la homogeneidad de las submuestras, pues al inicio cuando la mezcla está más caliente, el ligante asfáltico se adhiere a las paredes con la consiguiente pérdida del material (aún cuando indica que hay que utilizar un antiadherente) y cuando la muestra se enfría este fenómeno no se presenta pero la mezcla se aglomera en las partes finas lo que hace que el cuarteo se vea afectado.

Los cuarteadores pequeños no sirven para el tamaño de muestras que normalmente se muestrean que son de un tamaño de $21 \mathrm{~kg}$, pues no cabe en la bandeja del cuarteador. Por lo que no es posible utilizar este método para muestras de gran tamaño.

Los métodos de reducción tipo incremental tienen el problema que indican que se tiene que hacer un acomodo de la mezcla en un apilamiento tipo cónico, lo que hace que el agregado grueso se separe y produce una clara segregación de la muestra a pesar que el mismo método indica que se tiene que evitar pero los agregados de gran tamaño y peso siempre van a tender a segregarse sobre todo en mezclas asfálticas de graduación abierta.

Es de interés que este método sea aplicado en otros laboratorios de autocontrol, control y verificación de la calidad de la mezcla asfáltica en caliente a nivel nacional para uniformizar los métodos más que están demostradas las debilidades del método AASHTO.

\section{BIBLIOGRAFÍA}

American Association of State Highway and Transportation Officials, AASHTO R 47 "Reducing samples of hot mix asphalt (HMA) to testing size". Standard Specifications for Transportation Materials and Methods of Sampling and Testing. XXXII ed., AASHTO, EUA, 2012.

American Association of State Highway and Transportation Officials, AASHTO T 248 "Reducing field samples of aggregate to testing size". Standard Specifications for Transportation Materials and Methods of Sampling and Testing. XXXII ed., AASHTO, EUA, 2012.

American Society for Testing and Materials, ASTM C 702 "Standard Practice for reducing samples of aggregate to test size”. ASTM, EUA, 2015.

Nebraska Department of Roads, Standard Method T 248, "Reducing field samples of aggregate to testing size". NDR, EUA, 2014.

Iowa Department of Transportation, "Method of preparation of bituminous mix samples for test especimens". IDT, EUA, 2004.

Colorado Department of Transportation, Procedure 55 "Reducing field samples of hot bituminous pavements to test size". CDT, EUA, 1997.

Asphalt Institute. The Asphalt Handbook. Manual Series №44, Edición 2007, EUA. 